\title{
Radiomics score predicts acute respiratory distress syndrome based on the initial CT scan after trauma
}

\author{
Sebastian Röhrich ${ }^{1} \cdot$ Johannes Hofmanninger ${ }^{2} \cdot$ Lukas Negrin $^{3} \cdot$ Georg Langs $^{2}$ (i) $\cdot$ Helmut Prosch $^{1}$
}

Received: 22 May 2020 / Revised: 2 December 2020 / Accepted: 16 December 2020 / Published online: 17 March 2021

(C) The Author(s) 2021, corrected publication 2021

\begin{abstract}
Objectives Acute respiratory distress syndrome (ARDS) constitutes a major factor determining the clinical outcome in polytraumatized patients. Early prediction of ARDS is crucial for timely supportive therapy to reduce morbidity and mortality. The objective of this study was to develop and test a machine learning-based method for the early prediction of ARDS derived from the first computed tomography scan of polytraumatized patients after admission to the hospital.

Materials and methods One hundred twenty-three patients (86 male and 37 female, age $41.2 \pm 16.4$ ) with an injury severity score (ISS) of 16 or higher $(31.9 \pm 10.9)$ were prospectively included and received a CT scan within $1 \mathrm{~h}$ after the accident. The lungs, including air pockets and pleural effusions, were automatically segmented using a deep learning-based algorithm. Subsequently, we extracted radiomics features from within the lung and trained an ensemble of gradient boosted trees (GBT) to predict future ARDS. Results Cross-validated ARDS prediction resulted in an area under the curve (AUC) of 0.79 for the radiomics score compared to 0.66 for ISS, and 0.68 for the abbreviated injury score of the thorax (AIS-thorax). Prediction using the radiomics score yielded an f1-score of 0.70 compared to 0.53 for ISS and 0.57 for AIS-thorax. The radiomics score achieved a sensitivity and specificity of 0.80 and 0.76 .

Conclusions This study proposes a radiomics-based algorithm for the prediction of ARDS in polytraumatized patients at the time of admission to hospital with an accuracy that competes and surpasses conventional scores despite the heterogeneous, and therefore more realistic, scanning protocols.

Key Points

- Early prediction of acute respiratory distress syndrome in polytraumatized patients is possible, even when using heterogenous data.

- Radiomics-based prediction resulted in an area under the curve of 0.79 compared to 0.66 for the injury severity score, and 0.68 for the abbreviated injury score of the thorax.

- Highlighting the most relevant lung regions for prediction facilitates the understanding of machine learning-based prediction.
\end{abstract}

Keywords Thoracic injuries - Acute respiratory distress syndrome Polytrauma Multidetector computed tomography · Radiomics

Sebastian Röhrich and Johannes Hofmanninger have contributed equally in the creation of this manuscript and should be listed as shared firstauthors.

\section{Georg Langs}

georg.langs@meduniwien.ac.at

1 Department of Biomedical Imaging and Image-guided Therapy, Medical University of Vienna, Vienna, Austria

2 Computational Imaging Research Lab, Department of Biomedical Imaging and Image-Guided Therapy, Medical University of Vienna, Waehringer Guertel 18-20, A-1090 Vienna, Austria

3 Department of Orthopedics and Trauma-Surgery, Medical University of Vienna, Vienna, Austria

$\begin{array}{ll}\text { Abbreviations } \\ \text { AIS-thorax } & \text { Abbreviated injury score of the thorax } \\ \text { ARDS } & \text { Acute respiratory distress syndrome } \\ \text { AUC } & \text { Area under the curve } \\ \text { CNN } & \text { Convolutional neural network (CNN) } \\ \text { CT } & \text { Computed tomography } \\ \text { GBT } & \text { Gradient boosted trees } \\ \text { HU } & \text { Hounsfield units } \\ \text { ICU } & \text { Intensive care unit } \\ \text { ISS } & \text { Injury severity score } \\ \text { TTS } & \text { Thoracic trauma severity }\end{array}$




\section{Introduction}

Blunt thoracic trauma is a common injury mechanism in polytraumatized patients and of those, two-thirds may suffer from parenchymal lung injury [1]. The relevance of parenchymal injuries (lung contusion and laceration) lies in the increased risk of developing acute respiratory distress syndrome (ARDS) and, consequently, the resulting deterioration of clinical outcome [2-5]. Whereas lung contusion and the resulting damage of the alveolar epithelial cells are a direct factor in the genesis of ARDS [6-8], systemic inflammatory response mediators are important indirect pathomechanisms [9]. Predictive factors at admission to the hospital after blunt trauma for the development of ARDS comprise parenchymal lung injuries such as lung contusions, hypotension, necessity of blood transfusion, age higher than 65 years, and an injury severity score (ISS) higher than $25[10,11]$. Depending on the specifics of the study cohort, incidence and mortality may range from 5.8 to $21.5 \%$ and 12.2 to $24.9 \%$ respectively, with a decrease of both incidence and mortality in the last years [12-17]. Furthermore, there is also a considerable socioeconomic impact due to impaired cognitive and physical function after convalescence [18]. Currently, the accuracy of early prediction methods has an area under the curve (AUC) of 0.72 to 0.82 for trauma score $[11,19]$ and 0.67 to 0.75 for computed tomography (CT) volumetric methods [20,21]. Early prediction of ARDS in polytraumatized patients may enable timely supportive therapy, such as adjusting ventilation for lung protection, restricting the administration of transfusions, or starting the administration of antibiotics [22, 23], and, thus, reduce complications or prevent the development of ARDS altogether.

CT of the chest is a widespread and essential tool in the primary diagnostic process of trauma patients after admission to the hospital [24]. The initial traumatic injury results in focal or diffuse alveolar hemorrhage [25] followed by a lung edema and interstitial alterations in the affected parenchyma [26]. While these imaging findings may prove difficult to interpret at the beginning, and only become more prominent in the ensuing 24 to $48 \mathrm{~h}$ [27], early information from the first CT scan would help to decide on the abovementioned pivotal therapeutic decisions. Furthermore, the patient's condition may prevent a transport to the CT scanner and multiple exposures to ionizing radiation of the often young population of polytraumatized patients carry an increased risk of inducing oncological disease in survivors. Therefore, novel methods to raise as much information as possible from the first scan after admission to the hospital are crucial.

The European Society of Radiology endorses the extraction of quantitative biomarkers from medical images that inform on disease detection, characterization, monitoring, and assessment of response to treatment [28]. One method to extract information from images that is not entirely accessible for the human eye is radiomics [29], for which an increasing number of applications emerge in non-oncologic chest CT [30].

It was the aim of this study to develop and test a radiomicsbased computational method for the early prediction of ARDS based on the initial CT scan in a cohort of prospectively included patients with chest trauma.

\section{Methods}

\section{Data collection and study population}

The imaging and clinical data acquired for this study were collected in the framework of a prospective study which aimed to evaluate manual volumetry of parenchymal lung injury in initial and follow-up CT scans to predict ARDS in polytraumatized patients [31]. In total, 123 of the previously reported patients were evaluated. The previous study reported on the usefulness of a follow-up chest CT scan for manual volumetry of lung parenchymal injuries compared to the initial scan at admission whereas the present study evaluated the possibility of machine learning-based prediction of ARDS in the initial scan.

The patients were included over a timeframe of 4 years. Inclusion criteria were 18 years or older, ISS of 16 or higher, direct transport to the study hospital (level I trauma center) with a $\mathrm{CT}$ scan within $1 \mathrm{~h}$ after the trauma, and admission to the intensive care unit. Informed consent was gained from the patient or legal representative. Exclusion criteria were death within $48 \mathrm{~h}$ (this was a methodological necessity of the original publication [31]), burning injury, oncological disease, and chronic inflammatory lung disease. The Berlin Definition [7] was used to define ARDS based on oxygenation, chest imaging, timing, and origin of edema. The CTindependent methods of establishing ARDS were partial pressure of arterial oxygen $\left(\mathrm{PaO}_{2}\right) \leq 300 \mathrm{~mm} \mathrm{Hg}$ with positive end-expiratory pressure (PEEP) or continuous positive airway pressure $\geq 5 \mathrm{~cm} \mathrm{H}_{2} \mathrm{O}$, respiratory failure not fully explained by cardiac failure or fluid overload, and a development within 1 week of the trauma.

One hundred sixteen patients received intravenously administered contrast agent; for 7 patients, the administration of contrast agent was not possible. In patients who were hemodynamically unstable when reaching the emergency room $(n=6)$, we performed an arterial phase with a field of view ranging from the skull base to the proximal femur (bolus tracking technique at the aortic arch with a threshold of 100 Hounsfield units (HU) and 10-s delay; $120 \mathrm{ml}$ in total with a flow of $4.5 \mathrm{ml} / \mathrm{s}$ for $31 \mathrm{~s}$ followed by $3.3 \mathrm{ml} / \mathrm{s}$ for $18 \mathrm{~s}$ ), followed by a venous phase over the abdomen. All other patients received a venous phase over the chest and abdomen $(120 \mathrm{ml}$ in total with a flow of $3 \mathrm{ml} / \mathrm{s}$ over $55 \mathrm{~s})$. 


\section{Machine learning algorithm for ARDS prediction}

Figure 1 gives an overview of the machine learning algorithm for the prediction of ARDS.

First, we segmented the lung with a convolutional neural network $(\mathrm{CNN})$ approach to automatically delineate the lung including pleural effusions and air pockets (pneumothorax) [32]. Visual inspection by a radiologist showed a good fit of the segmentations to the thoracic cavity, excluding the mediastinum.

After lung segmentation in all cases, we learned a visual vocabulary to represent the appearance variability in the study cohort. Specifically, we extracted $832 \mathrm{D}$ radiomics features on a grid from axial slices throughout the lung with $1-\mathrm{cm}$ distance between the locations and within a kernel diameter of $2.4 \mathrm{~cm}$.

For the extraction of radiomics data, we have relied on pyradiomics, an open-source python package for the extraction of radiomics features [28]. Note that the pyradiomics libraries are research tools and not approved for clinical use.
We calculated first-order statistics and features on gray level co-occurrence, gray level run length, gray level size zone, and gray level dependence matrices. To ensure comparable radiomics feature across scans with variable voxel sizes, the image volumes were resampled to a voxel resolution of $0.8 \times 0.8 \times 2 \mathrm{~mm}$ [33]; voxel intensities were clipped at -990 and $200 \mathrm{HU}$. The extraction of texture features relies on the reduction of image intensities to a reduced number of discrete values. We quantized intensities into 40 bins (30 HU bin-width) for the calculation of the texture matrices. In addition to visual features, we calculated reference locations for each feature vector with three spatial coordinates (1) anteriorposterior, (2) inferior-superior, and the (3) distance to the lung border. Radiomics feature vectors together with the reference coordinate randomly sampled from all cases $(N \approx$ 24600 spatio-visual vectors) were used to learn a spatiovisual vocabulary, i.e., the feature vectors were clustered into 20 classes using $\mathrm{k}$-means, each class describing a visual pattern.
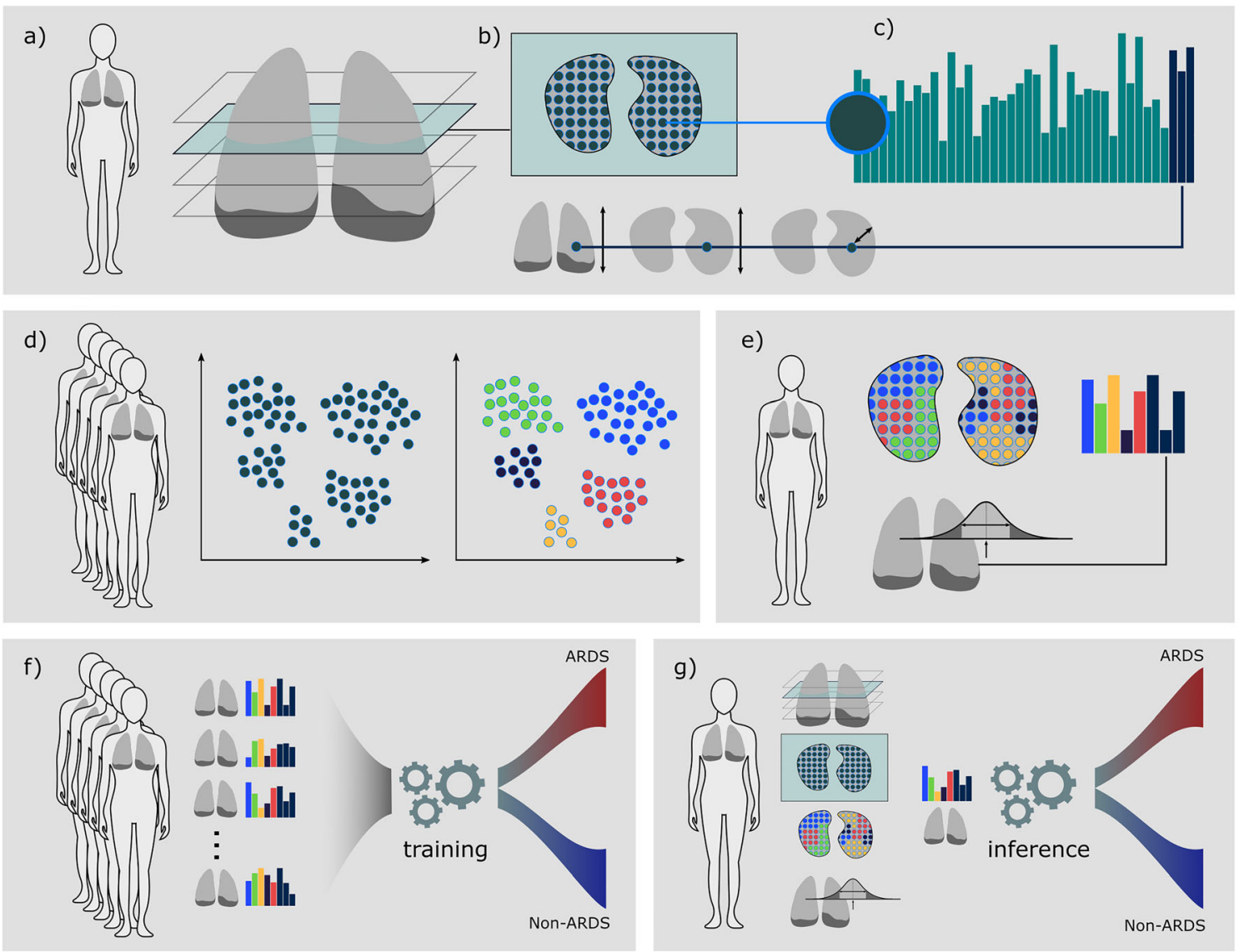

Fig. 1 Overview - from CT scan to ARDS prediction: a We performed a machine learning-based segmentation of the lung, including effusion and air pockets. b From within the lung-mask, we extracted 2D radiomics features in a grid pattern over a kernel. In addition, we calculated the reference locations (anterior-posterior, inferior-superior, and the distance transform) to retrieve localized spatio-visual feature vectors for each location as illustrated in c. d A spatio-visual vocabulary sampled from feature vectors of the full dataset was learned. e After the vocabulary

had been learned, a single patient is represented by his vocabulary histogram and statistical features calculated on the HU histogram of the full lung. f We trained a GBT ensemble on a training set of feature representations to distinguish cases that will develop ARDS in the future and cases that will not. g After training, prediction for a novel case is performed fully automated, from raw DICOM images, lung segmentation, and feature extraction to ARDS risk score. ARDS, acute respiratory distress syndrome; GBT, gradient boosted tree 
Subsequently, we calculated the vocabulary occurrence feature vectors for each single case. That is, the proportions of each of the vocabulary classes in a case form a quantized radiomics $(\mathrm{QR})$ feature vector capturing the composition of lung appearance patterns. In addition to these 20 values, we calculated 10 statistical features on all HU values within the lung: volume, mean, median, mean-average-deviation, variance, energy, $\%$ of $\mathrm{HU} \geq 300, \geq 200, \geq 100$, and $>0$.

Finally, we used these 30 lung features for machine learning-based prediction of whether the patient would develop ARDS or not by training a Gradient Boosted Tree (GBT) ensemble classifier [34] with $1 \mathrm{k}$ decision trees as provided by the scikit-learn library [35]. After training, ARDS risk prediction is performed fully automated, including lung segmentation and feature extraction without any human interaction required.

To estimate the predictive capabilities of the machine learning model on unseen data, we performed stratified k-fold cross-validation, a resampling procedure used to evaluate models on a limited data sample. In our study, 40 subsets of the original cases were obtained by randomization, and then every subset was validated against the remaining 39 subsets as training data, repeating this procedure 40 folds. Each fold in our study was composed of 39 ARDS cases, 81 (- 1) nonARDS in the training and one ARDS, $2(+1)$ non-ARDS cases in the test set. During each fold, the training data were used to optimize the model parameters while the test data were only used to report the prediction score.

The prediction performance was analyzed with a receiver operating characteristic (ROC) curve and additional prediction metrics (sensitivity, specificity, precision, and fl score) at the cutoffs yielded by the highest Youden index.

In addition to the supervised prediction experiments, we performed unsupervised cluster analysis based on the feature vectors. Statistically, a $\chi^{2}$ test was used to analyze the relationship between future ARDS status and the two main clusters formed by the radiomics feature expression. We report box plots and two-tailed $t$ tests to compare the non-ARDS and ARDS groups with respect to their risk scores (radiomics risk score, AIS, and ISS).

We performed statistical association tests to investigate potential spurious relationships between factors that may influence image appearance and future ARDS status. Specifically, we tested for associations between future ARDS and contrast agent administration, scanner model, slice thickness, reconstruction kernel, and dose. Furthermore, we calculated the evaluation scores (AUC, sensitivity, specificity, precision, and f1 score) for subsets of the population with homogeneous image parameters less influenced by these potential biases.

\section{Statistical analysis}

All statistical measures and tests were performed with the scipy statistics package (v 1.3.1) [36]. To test the associations between factors that may influence image appearance and future ARDS status, the Fisher exact tests have been performed for categorical variables (contrast agent administration, scanner model, slice thickness, and reconstruction kernel) and a two-tailed $t$ test has been performed for dose (mAs).

\section{Results}

In total, 123 patients with different blunt accident mechanisms (most common causes were pedestrian vs. vehicles (23.4\%), fall from $3 \mathrm{~m}$ or higher (25.9\%), and motor vehicle accidents $(38.8 \%))$ were included. Death occurred in five of the included patients (4\%), of whom three died from ARDS, one from multi-organ failure of different etiology, and one from brain injury. Detailed demographic characteristics of the study population are shown in Table 1, an overview of technical specifications of the CT scans is shown in Table 2, and thoracic injury patterns are listed in Table 3.

One hundred one (77.7\%) of the 123 patients had an abbreviated injury scale (AIS) of the thorax of 3 or higher, indicating a severe thoracic injury [37], of which 93 (71.5\% of 101) had a parenchymal lung injury.

To investigate feature expression patterns, we performed unsupervised clustering revealing groups of patients with similar feature patterns (Fig. 2). We compared the two main clusters of patients with their future ARDS status and found a significant association $\left(p=0.012, \chi^{2}\right.$ test).

Forty-fold cross-validation of ARDS prediction resulted in an AUC of 0.79 for the GBT-based radiomics score compared

Table 1 Characteristics of the study population

\begin{tabular}{ll}
\hline Patient characteristics & $\begin{array}{c}\text { Mean } \pm \text { Std; } \\
\text { number (\%) }\end{array}$ \\
\hline Male/female & $86 / 37$ \\
Age & $41.2 \pm 16.4$ \\
ISS & $31.9 \pm 10.9$ \\
AIS-thorax 0 & 11 \\
AIS-thorax 1 & 3 \\
AIS-thorax 2 & 13 \\
AIS-thorax 3 & 38 \\
AIS-thorax 4 & 33 \\
AIS-thorax 5 & 25 \\
Patients arriving intubated at the trauma center & $68(55.3 \%)$ \\
Patients with a chest tube insertion at the site of & $15(12.2 \%)$ \\
$\quad$ injury & $41(33.3 \%)$ \\
Patients with a chest tube insertion at the hospital & $3(2.4 \%)$ \\
Patients needing extracorporeal membrane & $40(32.5 \%)$ \\
$\quad$ oxygenation (ECMO) & \\
Patients developing acute respiratory distress & \\
$\quad$ syndrome (ARDS) &
\end{tabular}

ISS injury severity score, AIS-thorax abbreviated injury score of the thorax 
Table 2 Technical specifications of the CT scans

\begin{tabular}{llr}
\hline & Value & Number of cases \\
\hline Slice thickness (mm) & 1.5 & 38 \\
& 2.0 & 37 \\
& 3.0 & 41 \\
& 5.0 & 7 \\
In-plane pixel spacing (mm) & $0.56 \times 0.56$ to $0.98 \times 0.98$ & 123 \\
Tube voltage (kV) & 120 & 122 \\
Exposure (mAs) & 140 & 1 \\
Scanner & 39 to 467 & 123 \\
& Sensation 16 & 3 \\
& Sensation 4 & 11 \\
& Sensation Cardiac 64 & 6 \\
Convolution kernel & Sensation Open & 102 \\
& SOMATOM Definition & 1 \\
& B60s & 3 \\
& B60f & 5 \\
Contrast phase & B70s & 1 \\
& B70f & 114 \\
& Non-contrast & 7 \\
& Arterial & 6 \\
& Venous & 110 \\
& &
\end{tabular}

$m m$ millimeter, $k V$ kilovolt, $m A s$ milliampere second

to 0.66 for ISS, and 0.68 for the AIS-thorax score (Fig. 3). Additional prediction metrics such as sensitivity, specificity, precision, and f1 score can be found in Table 4 (cutoffs at the highest Youden index are plotted in Fig. 3). Prediction using the radiomics score yielded an $\mathrm{f} 1$ score of 0.70 compared to 0.53 for ISS and 0.57 for AIS-thorax. The radiomics score achieved a sensitivity/specificity of $0.80 / 0.76$. The features with the highest relative importance for classification yielded by the GBT ensemble during training were quantized radiomics feature 17 (QR17), $\mathrm{HU}>0$, and variance, with $\mathrm{HU}>0$ and variance showing positive correlation with future ARDS and QR17 showing a negative correlation. In Fig. 4, we illustrate the most relevant features $\mathrm{HU}>0$ and QR17 in cases with a high radiomics risk score for ARDS and cases with a low risk score.

Table 5 lists the relationships between factors that may influence image appearance and future ARDS status. The results indicate that the correlation between the scanner model and slice thickness with future ARDS status may not likely have occurred randomly. To assess the results less influenced by these potential biases, we performed an evaluation of the prediction model on two subgroups with homogeneous parameters: (subgroup 1) only cases scanned during the venous phase, scanner model S5 (see Table 5) and a high-frequency reconstruction algorithm (kernel B70f) and (subgroup 2) only
Table 3 Thoracic injury patterns in the study population

\begin{tabular}{|c|c|c|c|}
\hline Pathology & Location & $\begin{array}{l}\text { Frequency } \\
\text { per side }\end{array}$ & $\begin{array}{l}\text { Frequency in } \\
\text { total }\end{array}$ \\
\hline Lung contusion & $\begin{array}{l}\text { Unilateral } \\
\text { Bilateral }\end{array}$ & $\begin{array}{l}51(41.5 \%) \\
31(25.2 \%)\end{array}$ & $82(66.7 \%)$ \\
\hline Lung laceration & $\begin{array}{l}\text { Unilateral } \\
\text { Bilateral }\end{array}$ & $\begin{array}{c}21(17.1 \%) \\
1(0.8 \%)\end{array}$ & $22(17.9 \%)$ \\
\hline Pneumothorax & $\begin{array}{l}\text { Unilateral } \\
\text { Bilateral }\end{array}$ & $\begin{array}{c}43(35.0 \%) \\
6(4.9 \%)\end{array}$ & $49(39.8 \%)$ \\
\hline Hematothorax & $\begin{array}{l}\text { Unilateral } \\
\text { Bilateral }\end{array}$ & $\begin{array}{l}7(5.7 \%) \\
2(1.6 \%)\end{array}$ & $9(7.3 \%)$ \\
\hline Hematopneumothorax & $\begin{array}{l}\text { Unilateral } \\
\text { Bilateral }\end{array}$ & $\begin{array}{l}7(5.7 \%) \\
1(0.8 \%)\end{array}$ & $8(6.5 \%)$ \\
\hline Rib fractures & $\begin{array}{l}1 \mathrm{rib} \\
2 \mathrm{ribs} \\
\geq 3 \mathrm{ribs}\end{array}$ & $\begin{array}{c}16(13.0 \%) \\
7(5.7 \%) \\
64(52.0 \%)\end{array}$ & $87(70.7 \%)$ \\
\hline \multicolumn{3}{|l|}{ Flail chest } & $21(17.1 \%)$ \\
\hline \multicolumn{3}{|l|}{ Sternum fracture } & $25(20.3 \%)$ \\
\hline \multicolumn{3}{|l|}{ Thoracic spine fracture } & $37(30.1 \%)$ \\
\hline \multicolumn{3}{|c|}{ Extensive surgical emphysema } & $21(17.1 \%)$ \\
\hline \multicolumn{3}{|l|}{ Aortic dissection } & $7(5.7 \%)$ \\
\hline \multicolumn{3}{|l|}{ Diaphragmatic rupture } & $2(1.6 \%)$ \\
\hline \multicolumn{3}{|l|}{ Pneumomediastinum } & $6(4.9 \%)$ \\
\hline
\end{tabular}

cases with either slice thickness $2 \mathrm{~mm}$ or $3 \mathrm{~mm}$. The results show that the model remains predictive on a level nearly the same as the whole dataset, indicating a negligible influence of the tested factors with an $\mathrm{f} 1$ score of 0.70 for the full population, 0.67 for subgroup 1, and 0.69 for subgroup 2 (also see Table 4).

\section{Discussion}

The early recognition of a developing ARDS in the hours and days after trauma is critical for timely initiation of adequate treatment (such as adjusting ventilation for lung protection, preventing aspiration, restricting the administration of transfusions, or administration of antibiotics) which may decrease the incidence rate of full ARDS $[22,23]$. In the present study, we could demonstrate that a fully automated machine learning- and radiomics-based approach can identify polytraumatized patients with an increased risk of developing ARDS with higher accuracy than established scores by utilizing the first CT scan at the time of admission.

Currently, trauma scores such as the ISS or thoracic trauma severity (TTS) score can be used to estimate the risk of delayed ARDS after trauma [11, 19, 38]. However, the ISS is a combined score of different body regions and, thus, is prone to error due to the lack of weighting of different regions. With the proposed radiomics-based approach, a more specific 


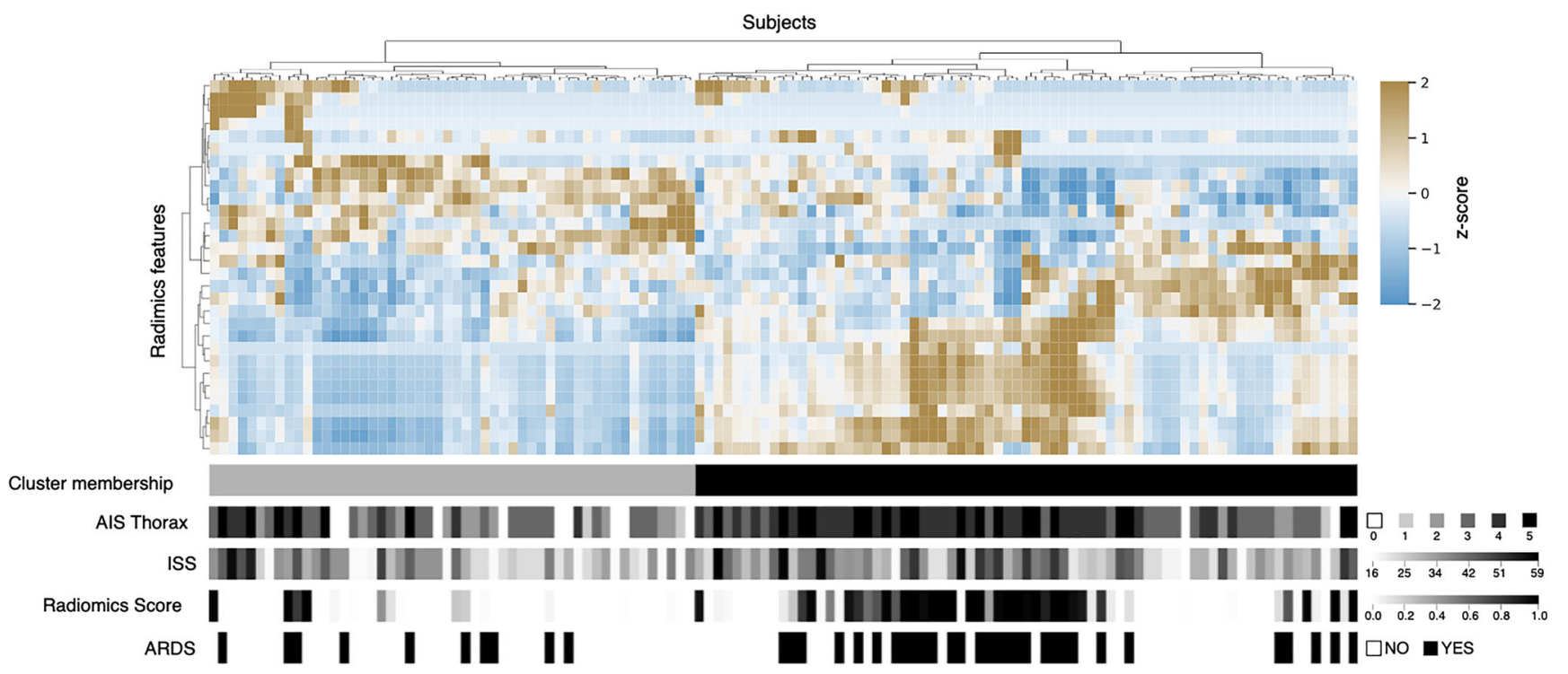

Fig. 2 Feature heatmap and unsupervised clustering: Clustered feature expressions and their association with injury severity scores, the machine learning-based radiomics risk score, and the future ARDS status. Each column represents one patient sorted after agglomerative clustering. A $\chi^{2}$

test reveals a significant relationship $(p=0.012)$ between the two main clusters formed by the radiomics feature expressions and the future ARDS status

representation of the lung's injuries could be achieved. Indeed, the accuracies for the prediction of ARDS are at a similar level or higher than what was achieved in several studies using trauma scores (this study: AUC of 0.79, ISS: AUC of 0.72 [11], TTS: AUC of 0.82 [19]). However, another study could show better results for the ISS with an AUC of 0.88 [12]. Additionally, the ISS score in our study showed markedly worse performance (AUC of 0.66 ) than the ISS scores in all of the aforementioned studies. A possible reason can be found in the different inclusion criteria: whereas the previous study included all patients regardless of ISS score, our study only included patients with an ISS score of 16 or higher. In a cohort that includes low ISS scores, the performance in predicting ARDS will be higher

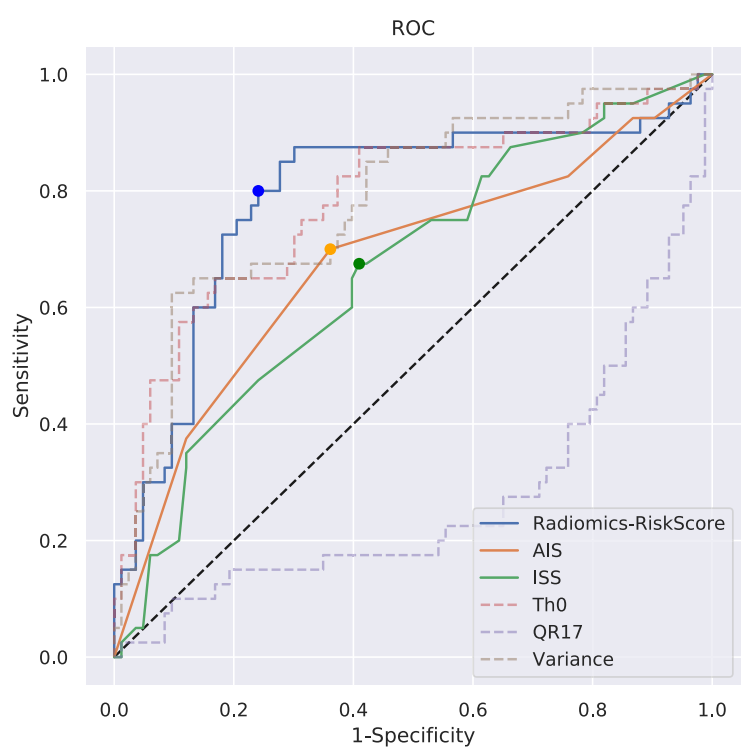

Fig. 3 Quantitative results: This receiver operating characteristic (ROC) curve shows the superior performance of radiomics-based prediction of acute respiratory distress syndrome (ARDS) compared to conventional trauma scores. In addition, the ROC curves for the three most relevant features as reported by the GBT ensemble are shown and the scores and
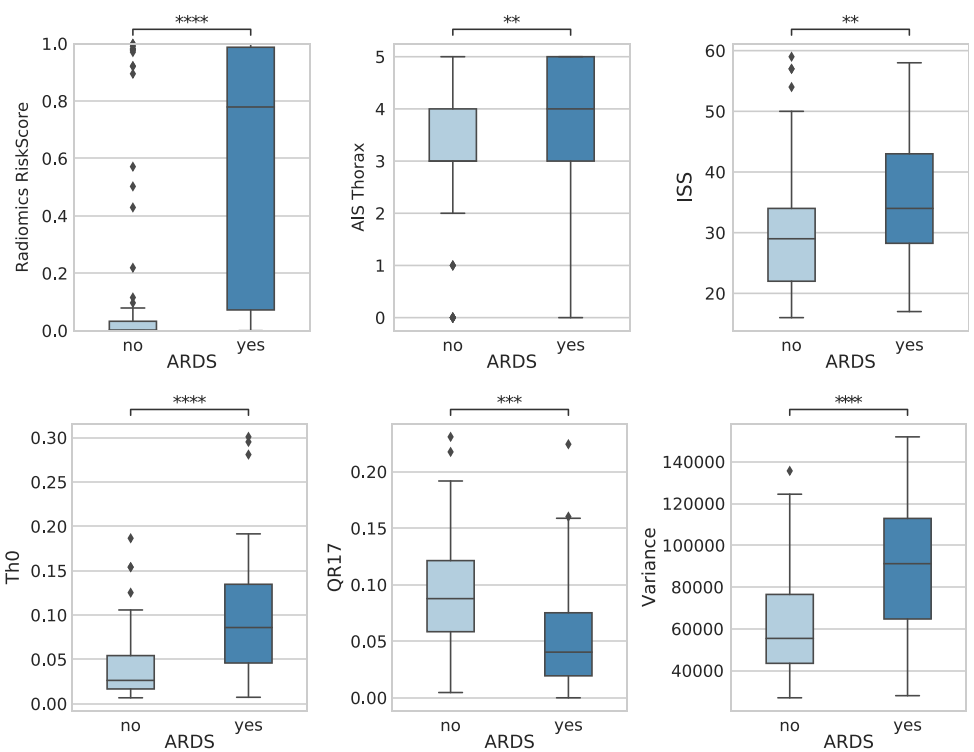

feature expressions are compared between ARDS and non-ARDS cases via boxplots and $t$ tests. $* *: 1.00 \mathrm{e}-03<p<=1.00 \mathrm{e}-02$; $* * *: 1.00 \mathrm{e}-04<$ $p<=1.00 \mathrm{e}-03$; $* * * *: p<=1.00 \mathrm{e}-04$. ISS, injury severity score; AIS, abbreviated injury scale (for thorax) 
Table 4 Results for the prediction of acute respiratory distress syndrome

\begin{tabular}{|c|c|c|c|c|c|}
\hline Prediction based on & Sensitivity & Specificity & Precision & f1 score & AUC \\
\hline ISS & 0.68 & 0.59 & 0.44 & 0.53 & 0.66 \\
\hline AIS & 0.70 & 0.64 & 0.48 & 0.57 & 0.68 \\
\hline Radiomics score (whole dataset) & 0.80 & 0.76 & 0.62 & 0.70 & 0.79 \\
\hline Radiomics score (subgroup 1) & 0.81 & 0.79 & 0.57 & 0.67 & 0.79 \\
\hline Radiomics score (subgroup 2) & 0.81 & 0.71 & 0.59 & 0.69 & 0.75 \\
\hline Rib fractures (yes/no) & 0.75 & 0.31 & 0.34 & 0.47 & \\
\hline
\end{tabular}

ISS injury severity score, AIS abbreviated injury scale (for thorax), AUC area under the curve, subgroup 1 only cases scanned during a venous phase, scanner model S5, and kernel B70f, subgroup 2 only cases with 2-mm or 3-mm slice thickness

as low ISS scores will lead to more true-negative cases [12]. In our study, the accuracy is reduced by patients that are more heavily injured, but not injured enough to easily predict the occurrence of ARDS. Thus, a radiomics-based approach may help in further stratifying a more heavily injured patient cohort for which the ISS score has reduced predictive capabilities.

One reason for this may be the importance of direct lung injury as an independent risk factor for the development of

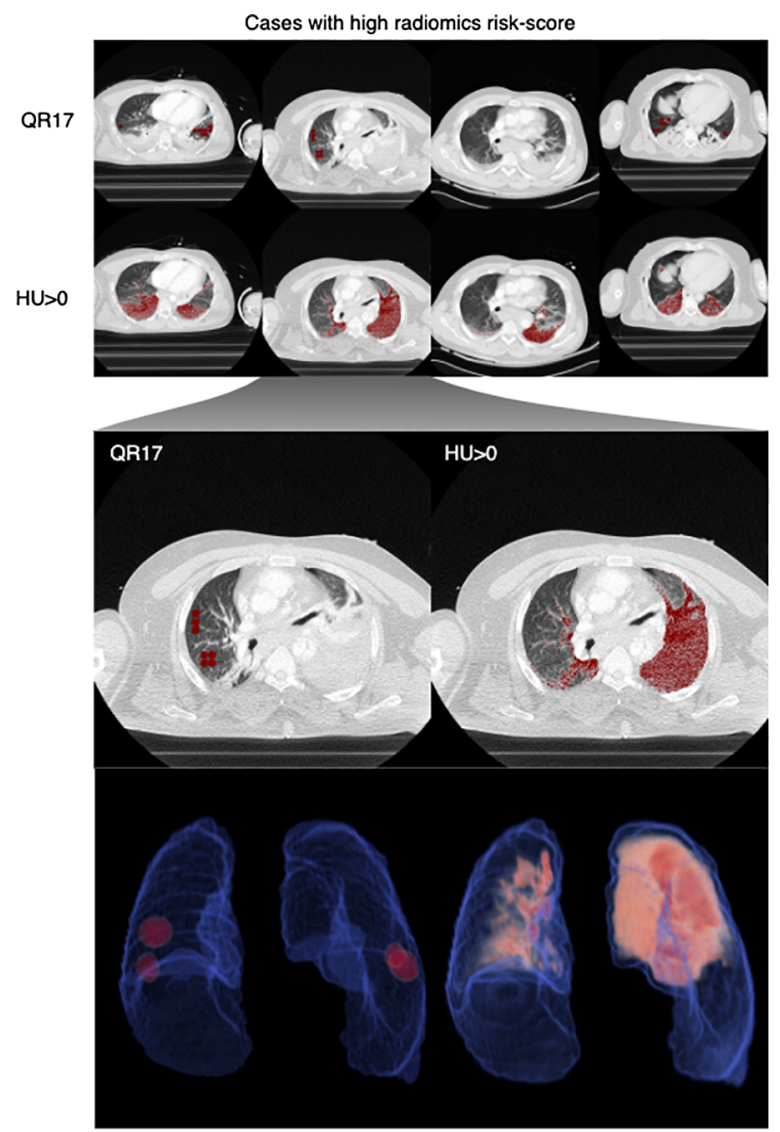

Fig. 4 Visualization of predictive features: Here, the two most relevant visual features for ARDS prediction (QR17 and HU $>0)$ as reported by the GBT ensemble are visualized. On the left side, the four cases which
ARDS, in particular forms of parenchymal lung injuries such as lung contusions or lacerations [11]. Parenchymal lung injuries cause localized and generalized inflammatory reactions that may lead to ARDS $[39,40]$. Consequently, both studies involving $\mathrm{CT}$ and ultrasound have found a positive correlation of the extent of lung contusions with the development of ARDS [20, 21, 31, 41, 42].

However, manual volumetry of parenchymal lung injuries is a very time-consuming task and not fit for clinical

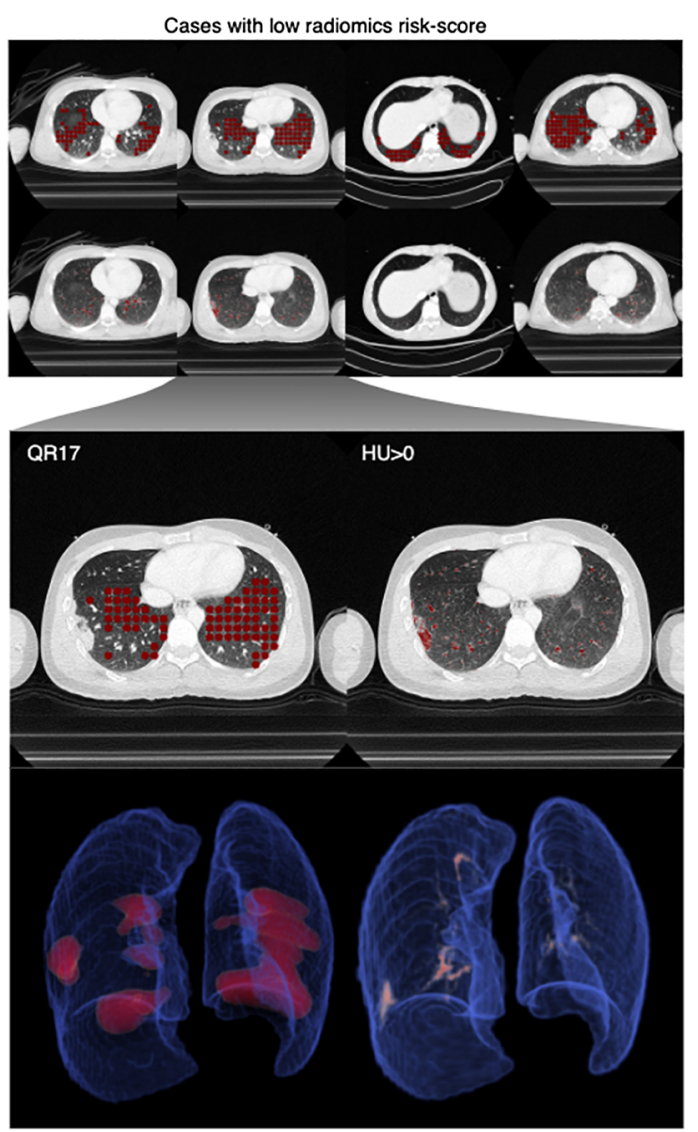

received the highest risk score are shown and on the right side the four cases which received the lowest risk score. In addition, a $3 \mathrm{D}$ visualization of the locations of the features is shown in two representative cases 
Table 5 Assessment of potential technical biases

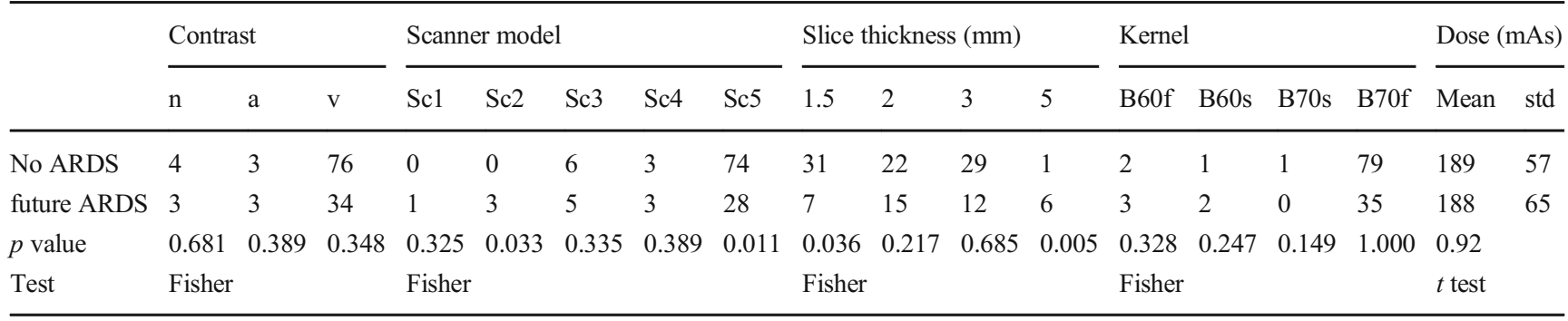

ARDS acute respiratory distress syndrome, $n$ no contrast, $a$ arterial phase; $v$ venous phase, $S c 1$ SOMATOM Definition, $S c 2$ Sensation $16, S c 3$ Sensation 4, Sc4 Sensation Cardiac 64, Sc5 Sensation Open, $m A s$ milliampere second, std standard deviation, Fisher Fisher exact test

application, especially in the time-critical setting of trauma care. Finally, disregarding other pulmonary or pleural pathologies after trauma (e.g., hemothorax) could reduce the accuracy for predicting ARDS. A study investigating chest injury patterns after blunt trauma has shown pleural collections to be present in $\sim 30 \%$ of all cases and in $83 \%$ of fatal cases [43]. A hemothorax is important for the prognosis of morbidity or mortality after trauma in two ways: on the one hand, by indicating bleeding from lung laceration or due to vessel injury and, on the other hand, by constituting a risk factor for the development of restrictive and infectious pleural processes, and consequently, respiratory failure [44]. Considering the relevance of a hemothorax, we included pleural effusions as part of the lung segmentations. The visualization of predictive features (see Fig. 4) suggests a high relevance for increased densities in the posterior pleural space, attributable to hemothorax, and for increased lung parenchymal densities, such as in parenchymal lung injuries.

Increased lung densities can represent one or several underlying pathologies (i.e., parenchymal lung injuries, edema, bleeding from another lung region, atelectasis). A clear statement about the densities' etiology from images alone is often not possible for radiologists. Consequently, a study that applied a semiautomatic method based on thresholds of elevated lung density resulted in a better prediction of ARDS occurrence than human readers [45]. The advantage of a radiomics-based approach is the possibility of providing additional information beyond what human vision is capable of by not only assessing density thresholds, but by including complex statistical relationships of voxels [29]. Feature importance analysis showed that a generic threshold (HU $>0$ ) has high relevance and a positive association with future ARDS status. However, one of the fine-grained appearance classes (QR17) showed high relevance and a negative association. Visual inspection indicates that this feature encodes a form of inconspicuous parenchyma texture.
In trauma, both pulmonary and extrapulmonary risk factors influence the course of ARDS development, and, with an accuracy of $71 \%$, it is possible to assign an extrapulmonary origin to a typical, more symmetric pattern of ARDS in chest CT [46]. It would be interesting to assess the performance of a radiomics analysis to discriminate between ARDS of pulmonary and extrapulmonary origin. Regarding the prediction of ARDS based on lung alterations that occur during or immediately after the trauma, it seems unlikely that our proposed approach can reliably predict ARDS of extrapulmonary origin as CT images of lung parenchymal changes are the sole input of the method.

One known extrapulmonary predictor of ARDS is rib fracture [47]. Whereas our method focused on a radiomics-based approach to extract information from parenchymal and pleural pathologies, adding rib fractures as a categorical or ordinal variable might increase the accuracy of ARDS prediction. Automated rib fracture detection on a per case basis (i.e., are rib fractures present or not in a patient) already exists and could be considered an addition in further research [48]. Extrapulmonary predictors, such as cardiopulmonary or hematologic disease [47] or administration of fluids and transfusions [11], provide further relevant information. However, the clinical utility is hampered as in trauma patients it may be impossible to raise a comprehensive history or clinical parameters may only be available later on. Still, relevant information for the prediction of ARDS can be extracted from the electronic health record within the first $6 \mathrm{~h}$ after admission to the hospital by using a machine learning-based approach [49].

In addition to a risk estimation in an acute setting, a radiomics-based lung score could also be utilized for monitoring intensive care unit (ICU) patients. The complex course of disease progression and extensive data of ICU patients render the correct interpretation of a patient's state a challenging task for clinicians. A recent study has shown the possibility of integrating various clinical parameters by a machine learning-based algorithm to predict complications (i.e., bleeding, renal 
failure, and mortality) of ICU patients at a level higher than conventional clinical risk models [50]. Such algorithms have the potential to take a pivotal supportive role in prediction, diagnosis, and monitoring of ICU patients and, furthermore, are expandable by adding other forms of data. Whereas a conventional model may struggle incorporating laboratory data and vital functions with medical imaging information, a machine learning model is able to include radiomics features as additional data.

We recognize several limitations of this study. We excluded patients with an ISS of 15 or lower; therefore, we can only assess the predictive capabilities of the proposed algorithm in a collective with major injuries and an increased risk of ARDS. However, the relatively low predictive value of ISS scores in this study's dataset of polytraumatized patients compared to collectives that including less severe injuries (0.66 vs. 0.72 in [11]) indicates a more challenging setting. Furthermore, our cohort may be biased toward "late" ARDS (occurring $>48 \mathrm{~h}$ after trauma) compared to "early" ARDS $(<48 \mathrm{~h})$ due to the exclusion of patients who died within $48 \mathrm{~h}$.

To eliminate possible biases through heterogeneous scanner parameters, a completely standardized protocol on the same scanner would be preferential. In real clinical situations, particularly across different institutions, such preconditions are rarely met. Some of the scanner parameters that may influence radiomics features in this study are different flow rates and time after contrast agent administration [51], as well as reconstruction kernel, dose, slice thickness, and scanner type [52]. This is relevant because a machine learning model might learn to differentiate patients within the study population based on technical features, rather than the pathological features. In this study, 3 of 7 (43\%) patients with a non-contrast CT, 3 of $6(50 \%)$ patients with an arterial phase CT, and 34 of $110(31 \%)$ patients with a venous phase CT developed ARDS. We tested for such potential biases, assessed the predictive performance in a more homogeneous subgroup, and came to the conclusion that the influence on the model's predictive performance is negligible in our dataset (see the "Results" section, Tables 4 and 5). Interestingly, a correlation between a specific scanner and future ARDS occurrence may be due to the fact that more severely injured patients get transferred to a different scanner when the primary emergency room scanner is occupied, whereas less severely injured patients are kept on hold until the primary scanner is vacant again. Such organizational specifics should be kept in mind as a potential selection bias.

The features with the highest predictive capability were markedly increased densities in the posterior region of the thorax. While the timing of acquisition after injection of contrast agent may lead to a diffuse increase in $\mathrm{HU}$ of the lung, which theoretically might be learned by the algorithm, the visualization of relevant features suggests that the prediction of ARDS was based on regional abnormalities, rather than the systematic enhancement.

Furthermore, a standardization of inspiration depth is not possible for patients who experience severe chest pain or those who are intubated and with critical vital parameters, making respiration-based changes in lung density another potential source of noise. In the same manner, the elapsed time between trauma and CT scan may lead to alterations in lung densities as these may change rapidly depending on their etiology (e.g., bleeding, edema, atelectasis). In this study, all scans were conducted within one hour after the trauma.

Generally, a radiomics-based algorithm is agnostic towards predefined concepts of biology and pathology. Therefore, patterns and relationships discovered by such an approach are not directly attributable to common radiological patterns. However, they facilitate hypothesis generation for future studies through the discovery of novel patterns and their visual or statistical relationships.

In summary, we propose a radiomics-based algorithm for the prediction of ARDS in polytraumatized patients at the time of admission to hospital with an accuracy that competes and surpasses conventional scores despite the heterogeneous, and therefore more realistic, scanning protocols. Due to the generic nature of radiomics features, this algorithm may constitute a foundation for future, more complex models that integrate medical imaging information with clinical parameters. Furthermore, patterns identified as predictive signatures for ARDS may serve as a basis for hypotheses regarding underlying biological mechanisms.

Funding Open Access funding provided by Medical University of Vienna.

\section{Compliance with ethical standards}

Guarantor The scientific guarantor of this publication is Prof. Dr. Helmut Prosch.

Conflict of interest The authors of this manuscript declare relationships with the following companies: S.R.: Consulting activities for contextflow GmbH; J.H.: Speaker fees: Boehringer-Ingelheim; L.N.: no relationships with any companies, whose products or services may be related to the subject matter of the article; G.L.: Shareholder/Co-Founder contextflow GmbH; Speaker fees: Roche, Siemens; H.P.: Speakers fees: BoehringerIngelheim, Roche, Novartis, MSD, BMS, GSK, Chiesi, AstraZeneca.

Statistics and biometry One of the authors has significant statistical expertise.

Informed consent Written informed consent was obtained from all patients in this study.

Ethical approval Institutional Review Board approval was obtained.

Study subjects or cohorts overlap The imaging and clinical data acquired for this study were collected in the framework of a prospective 
study which aimed to evaluate manual volumetry of parenchymal lung injury in initial and follow-up CT scans to predict ARDS in polytraumatized patients (Title: "The clinical benefit of a follow-up thoracic computed tomography scan regarding parenchymal lung injury and acute respiratory distress syndrome in polytraumatized patients"); please also see the attached PDF. The previous study reported on the usefulness of a follow-up chest CT scan for manual volumetry of lung parenchymal injuries compared to the initial scan at admission whereas the present study evaluated the possibility of deep learning-based prediction of ARDS in the initial scan.

\section{Methodology \\ - prospective \\ - diagnostic or prognostic study \\ - performed at one institution}

Open Access This article is licensed under a Creative Commons Attribution 4.0 International License, which permits use, sharing, adaptation, distribution and reproduction in any medium or format, as long as you give appropriate credit to the original author(s) and the source, provide a link to the Creative Commons licence, and indicate if changes were made. The images or other third party material in this article are included in the article's Creative Commons licence, unless indicated otherwise in a credit line to the material. If material is not included in the article's Creative Commons licence and your intended use is not permitted by statutory regulation or exceeds the permitted use, you will need to obtain permission directly from the copyright holder. To view a copy of this licence, visit http://creativecommons.org/licenses/by/4.0/.

\section{References}

1. Elmali M, Baydin A, Nural MS et al (2007) Lung parenchymal injury and its frequency in blunt thoracic trauma: the diagnostic value of chest radiography and thoracic CT. Diagn Interv Radiol 13:179-182

2. Matthay MA, Ware LB, Zimmerman GA (2012) The acute respiratory distress syndrome. J Clin Investig 122:2731-2740

3. Wu J, Sheng L, Ma Y et al (2008) The analysis of risk factors of impacting mortality rate in severe multiple trauma patients with posttraumatic acute respiratory distress syndrome. Am J Emerg Med 26:419-424

4. Alisha C, Gajanan G, Jyothi H (2015) Risk factors affecting the prognosis in patients with pulmonary contusion following chest trauma. J Clin Diagn Res 9:OC17-OC19

5. Neher MD, Weckbach S, Flierl MA et al (2011) Molecular mechanisms of inflammation and tissue injury after major trauma-is complement the "bad guy"? J Biomed Sci 18:90

6. Perl M, Lomas-Neira J, Venet F et al (2011) Pathogenesis of indirect (secondary) acute lung injury. Expert Rev Respir Med 5:115126

7. ARDS Definition Task Force, Ranieri VM, Rubenfeld GD et al (2012) Acute respiratory distress syndrome: the Berlin Definition. JAMA 307:2526-2533

8. Calfee CS, Janz DR, Bernard GR et al (2015) Distinct molecular phenotypes of direct vs indirect ARDS in single-center and multicenter studies. Chest 147:1539-1548

9. Garcia CSNB, Pelosi P, Rocco PRM (2008) Pulmonary and extrapulmonary acute respiratory distress syndrome: are they different? Rev Bras Ter Intensiva 20:178-183

10. Baker SP, O’Neill B, Haddon W Jr, Long WB (1974) The injury severity score: a method for describing patients with multiple injuries and evaluating emergency care. J Trauma 14:187-196
11. Miller PR, Croce MA, Kilgo PD et al (2002) Acute respiratory distress syndrome in blunt trauma: identification of independent risk factors. Am Surg 68:845-850 discussion 850-1

12. Afshar M, Smith GS, Cooper RS et al (2016) Trauma indices for prediction of acute respiratory distress syndrome. J Surg Res 201: 394-401

13. Fahr M, Jones G, O’Neal H et al (2017) Acute respiratory distress syndrome incidence, but not mortality, has decreased nationwide: a National Trauma Data Bank Study. Am Surg 83:323-331

14. O'Leary MP, Keeley JA, Yule A et al (2016) Clinical predictors of early acute respiratory distress syndrome in trauma patients. Am J Surg 212:1096-1100

15. Daher P, Teixeira PG, Coopwood TB et al (2018) Mild to moderate to severe: what drives the severity of ARDS in trauma patients? Am Surg 84:808-812

16. Rubenfeld GD, Caldwell E, Peabody E et al (2005) Incidence and outcomes of acute lung injury. N Engl J Med 353:1685-1693

17. van Wessem KJP, Leenen LPH (2018) Incidence of acute respiratory distress syndrome and associated mortality in a polytrauma population. Trauma Surg Acute Care Open 3:e000232

18. Herridge MS, Tansey CM, Matté A et al (2011) Functional disability 5 years after acute respiratory distress syndrome. N Engl J Med 364:1293-1304

19. Daurat A, Millet I, Roustan J-P et al (2016) Thoracic Trauma Severity score on admission allows to determine the risk of delayed ARDS in trauma patients with pulmonary contusion. Injury 47 : $147-153$

20. Wang S, Ruan Z, Zhang J, Jin W (2011) The value of pulmonary contusion volume measurement with three-dimensional computed tomography in predicting acute respiratory distress syndrome development. Ann Thorac Surg 92:1977-1983

21. Strumwasser A, Chu E, Yeung L et al (2011) A novel CT volume index score correlates with outcomes in polytrauma patients with pulmonary contusion. J Surg Res 170:280-285

22. Beitler JR, Schoenfeld DA, Taylor Thompson B (2014) Preventing ARDS. Chest 146:1102-1113

23. Yilmaz M, Keegan MT, Iscimen R et al (2007) Toward the prevention of acute lung injury: protocol-guided limitation of large tidal volume ventilation and inappropriate transfusion. Crit Care Med 35:1660-1666

24. Johnson SB (2008) Tracheobronchial injury. Semin Thorac Cardiovasc Surg 20:52-57

25. Raghavendran K, Davidson BA, Woytash JA et al (2005) The evolution of isolated bilateral lung contusion from blunt chest trauma in rats: cellular and cytokine responses. Shock 24:132-138

26. Sangster GP, González-Beicos A, Carbo AI et al (2007) Blunt traumatic injuries of the lung parenchyma, pleura, thoracic wall, and intrathoracic airways: multidetector computer tomography imaging findings. Emerg Radiol 14:297-310

27. Cohn SM (1997) Pulmonary contusion: review of the clinical entity. J Trauma 42:973-979

28. deSouza NM, Achten E, Alberich-Bayarri A et al (2019) Validated imaging biomarkers as decision-making tools in clinical trials and routine practice: current status and recommendations from the EIBALL* subcommittee of the European Society of Radiology (ESR). Insights Imaging 10:87

29. Lambin P, Rios-Velazquez E, Leijenaar R et al (2012) Radiomics: extracting more information from medical images using advanced feature analysis. Eur J Cancer 48:441-446

30. Röhrich S, Hofmanninger J, Prayer F et al (2020) Prospects and challenges of radiomics by using nononcologic routine chest $\mathrm{CT}$. Radiology: Cardiothoracic Imaging 2:e190190

31. Negrin LL, Prosch H, Kettner S et al (2017) The clinical benefit of a follow-up thoracic computed tomography scan regarding parenchymal lung injury and acute respiratory distress syndrome in polytraumatized patients. J Crit Care 37:211-218 
32. Hofmanninger J, Prayer F, Pan J, Röhrich S, Prosch H, Langs G (2020) Automatic lung segmentation in routine imaging is primarily a data diversity problem, nota methodology problem. Eur Radiol Exp 4(1):50. https://doi.org/10.1186/s41747-020-00173-2

33. Shafiq-Ul-Hassan M, Zhang GG, Latifi K et al (2017) Intrinsic dependencies of CT radiomic features on voxel size and number of gray levels. Med Phys 44:1050-1062

34. Friedman JH (2001) Greedy function approximation: a gradient boosting machine. Ann Stat 29:1189-1232

35. Pedregosa F, Varoquaux G, Gramfort A et al (2011) Scikit-learn: machine learning in Python. J Mach Learn Res 12:2825-2830. https://scikit-learn.org/stable/index.html. Accessed 31 March 2020

36. Virtanen P, Gommers R, Oliphant TE et al (2020) SciPy 1.0: fundamental algorithms for scientific computing in Python. Nat Methods 17:261-272

37. Gennarelli TA, Wodzin E (2006) AIS 2005: a contemporary injury scale. Injury 37:1083-1091

38. Bayer J, Lefering R, Reinhardt S et al (2017) Thoracic trauma severity contributes to differences in intensive care therapy and mortality of severely injuredpatients: analysis based on the TraumaRegister DGU®. World J Emerg Surg 12:43. https://doi. org/10.1186/s13017-017-0154-1

39. Ciesla DJ, Moore EE, Johnson JL et al (2005) The role of the lung in postinjury multiple organ failure. Surgery 138:749-758

40. Raghavendran K, Notter RH, Davidson BA et al (2009) LUNG CONTUSION. Shock 32:122-130

41. Becher RD, Colonna AL, Enniss TM et al (2012) An innovative approach to predict the development of adult respiratory distress syndrome in patients with blunt trauma. J Trauma Acute Care Surg 73:1229-1235

42. Miller PR, Croce MA, Bee TK et al (2001) ARDS after pulmonary contusion: accurate measurement of contusion volume identifies high-risk patients. J Trauma 51:223-230
43. Galan G, Peñalver JC, París F et al (1992) Blunt chest injuries in 1696 patients. Eur J Cardiothorac Surg 6:284-287

44. Mowery NT, Gunter OL, Collier BR et al (2011) Practice management guidelines for management of hemothorax and occult pneumothorax. J Trauma 70:510-518

45. Daly M, Miller PR, Jeffrey Carr J et al (2008) Traumatic pulmonary pathology measured with computed tomography and a semiautomated analytic method. Clin Imaging 32:346-354

46. Desai SR, Wells AU, Suntharalingam G et al (2001) Acute respiratory distress syndrome caused by pulmonary and extrapulmonary injury: a comparative CT study. Radiology 218:689-693

47. Tignanelli CJ, Hemmila MR, Rogers MAM, Raghavendran K (2019) Nationwide cohort study of independent risk factors for acute respiratory distress syndrome after trauma. Trauma Surg Acute Care Open 4:e000249

48. Weikert T, Noordtzij LA, Bremerich J et al (2020) Assessment of a deep learning algorithm for the detection of rib fractures on wholebody trauma computed tomography. Korean J Radiol 21:891-899

49. Zeiberg D, Prahlad T, Nallamothu BK et al (2019) Machine learning for patient risk stratification for acute respiratory distress syndrome. PLoS One 14:e0214465

50. Meyer A, Zverinski D, Pfahringer B et al (2018) Machine learning for real-time prediction of complications in critical care: a retrospective study. Lancet Respir Med 6:905-914

51. Kim H, Park CM, Park SJ et al (2016) Temporal changes of texture features extracted from pulmonary nodules on dynamic contrastenhanced chest computed tomography. Invest Radiol 51:569-574

52. Mackin D, Fave X, Zhang L et al (2015) Measuring computed tomography scanner variability of radiomics features. Invest Radiol 50:757-765

Publisher's note Springer Nature remains neutral with regard to jurisdictional claims in published maps and institutional affiliations. 